\title{
Albumin As a Resuscitative Fluid in Patients with Severe Sepsis: A Randomized Clinical Trial
}

Farzad Kakaei ${ }^{1,2}$, Shahriar Hashemzadeh ${ }^{1}$, Ayyoub Asheghvatan ${ }^{1}$, Sina Zarrintan ${ }^{1 *}$, Touraj Asvadi ${ }^{1}$, Samad Beheshtirouy ${ }^{3}$, and Arian Mohajer ${ }^{4}$

1- Department of General Surgery, Faculty of Medicine, Tabriz University of Medical Sciences, Tabriz, Iran

2- Section of Visceral Transplatation, Imam Reza Hospital, Tabriz University of Medical Sciences, Tabriz, Iran

3- Department of Cardiothoracic Surgery, Imam Reza Hospital, Tabriz University of Medical Sciences, Tabriz, Iran

4- Faculty of Medicine, Tabriz University of Medical Sciences, Tabriz, Iran

\begin{abstract}
Fluid therapy is an essential part of resuscitation of the patients with severe sepsis and different methods has been suggested for. One of these methods is to add colloids like albumin to the crystalloid fluids, yet there are serious controversies about its effectiveness. To determine the effect of adding albumin to crystalloid fluids on the outcome of the severe sepsis, we performed this study. 20 patients ( 12 men and 8 women, with the mean age of 58) with severe sepsis who needed resuscitation, who were hospitalized in Imam Reza medical education center. The study began on march 2015 and ended on march 2016. The patients were randomized into two groups. One group received crystalloid fluids and routine management procedures and the other group received albumin (two vials of $20 \%$ albumin) alongside those two mentioned. Mortality was the primary endpoint. Secondary endpoints were the length of hospitalization, the need for mechanical ventilation, dialysis and organ failure. 28 and 90 day mortality in the albumin group was 10 and 40 percent, respectively ( $p$ value $=0.58$ ), and they were 30 and 50 percent in the control group, respectively ( $p$ value $=0.5$ ). None of the patients needed dialysis and there were no significant differences between the two groups from the organ failure point of view. The median length of hospitalization in both ICU and general ward was significantly higher in the albumin group (16 days VS 7.5 days, $p$ value $=0.03$ and 32 days VS 12 days, $\mathrm{p}$ value $=0.02$ ). $\mathrm{PaO}_{2}$ and $\mathrm{O}_{2}$ Sat were lower but not significant in the albumin group. Adding albumin to the crystalloid fluids in order to resuscitate the patients with severe sepsis, only results in prolongation of the hospitalization length in both ICU and general ward and has no notable clinical benefit.

Trial registration www.IRCT.org/ IRCT 2016040316473N5

Keywords: Albumin; Resuscitative Fluid; Sepsis; Randomized Clinical Trial
\end{abstract}

Corresponding author:

Sina Zarrintan

General Surgery, Sina Hospital, Tabriz University of Medical Sciences, Tabriz, Iran

E-mail: s.zarrintan@yahoo.com

Receive date: 2017-06-17 | Accept date: 2017-09-04 | Publish date: 2017-10-01

DOI: 10.7575/aiac.abcmed.17.05.04.02 


\section{Introduction}

Albumin is a $66 \mathrm{kDa}$ protein which contains 585 amino acids. It has a negative charge and is produced in the liver (10-15 $\mathrm{gr} /$ day) in response to the changes in colloid oncotic pressure ${ }^{[1]}$. Only 30-40 percent of albumin is in plasma and about $5 \%$ of it, leaves the vascular system and enters the interstitium [2]. Albumin plays a major role in creating the oncotic pressure, is a ligand for bilirubin, FFA and ions ${ }^{[1]}$. Albumin also has anti inflammatory effects, regulates the immune system and acts as an antioxidant which can be helpful in sepsis [3]. Crystalloid fluids are used commonly as fluid therapy in the patients with septic shock, but some authors suggest using albumin in these patients in order to maintain the oncotic pressure ${ }^{[4]}$. Those who agree with the administration of albumin in the patients with severe sepsis, believe that albumin can increase the volume in vascular system much faster and they also point to its possible role in eliminating the inflammatory mediators ${ }^{[5,6]}$. Some authors suggest that the administration of albumin in the patients with septic shock can reduce the mortality rate compared to the administration of the crystalloid fluids alone [7], while others suggest that the administration of albumin does not affect the mortality rate in the patients with septic shock ${ }^{[8]}$. Because of such controversies, we designed a pilot study in order to determine the efficacy of administration of the albumin alongside crystalloid fluids in patients with severe sepsis.

\section{Methods}

20 patients with severe sepsis who met the inclusion criteria written below, underwent randomization and divided into two groups. The inclusion criteria were: having at least 2 symptoms or signs of SIRS, having a confirmed source of infection and the need for fluid resuscitation. The exclusion criteria were: brain death, patients who underwent the plasmaphresis, any contraindications to the administration of albumin, organ failure (cardiac, pulmonary and renal) before sepsis, age under 12 years old, organ transplant, the history of cardiac surgery in the past three months, death in the first 24 hours and burn patients. The need fluid resuscitation was based on the presence of at least one of these criteria: tachycardia, systolic blood pressure less than $100 \mathrm{~mm} \mathrm{Hg}$, mean arterial pressure less than $75 \mathrm{~mm} \mathrm{Hg}$, urine output less than 5 $\mathrm{cc} / \mathrm{kg} / \mathrm{min}$, the need of vasopressors to control blood pressure and a CVP less than $10 \mathrm{mmHg}$. The first group received standard therapeutic measures for septic shock, such as crystalloid serum, antibiotics, vasopressors, cardiopulmonary support and appropriate surgical interventions. The second group received albumin in addition to all the measures administered for the first group. To do so, two vials of $20 \%$ albumin were administered in every 1 liter of patient's crystalloid serum. The transfusion of blood or blood products and starting enteric or IV feeding in both groups were based on the surgeon's opinion and had no relations to the kind of fluid therapy. The patients were followed up to 30 days or the day they expired at the hospital. The primary endpoint was mortality and the secondary endpoints were 31 day survival, degree of organ failures by SOFA criteria, length of hospitalization in ICU, level of need for mechanical ventilation and dialysis. SPSS $^{\text {TM }} 16$ used to analyze the data. The data are shown in as [IQR], median, (minimum- 
maximum) and frequency (\%). The comparison of the quantitative data was done using MannWhitney $U$ test and the comparison of the qualitative data was done with Chi-square or fisher's exact test. P-value under 0.05 was considered statistically significant.

\section{Results}

20 patients, 8 female and 12 male, who were diagnosed with severe sepsis, included in this study, With the median age of 58.5 years old (21-88 years old) in the albumin group and
57.5 years old (17-91 years old) in the first group and there was no significant difference between the two groups $(p=0.63)$. The median height of the albumin group was $171 \mathrm{~cm}$ (156$185 \mathrm{~cm})$ and was $170 \mathrm{~cm}(143-175 \mathrm{~cm})$ in the second group, there was no significant difference between the two groups $(p=0.68)$. $30 \%$ of the albumin group had sepsis because of peritonitis but this percentage was $40 \%$ in the first group. Other causes of sepsis are listed in table 1.

\begin{tabular}{lcc}
\hline Cause & First group & Second group \\
\hline Peritonitis & 40 & 30 \\
Liver abscess & 10 & 0 \\
Multi trauma & 30 & 20 \\
Esophageal & 20 & 10 \\
Cancer * & \\
Cholangitis & 0 & 20 \\
Fascitis** & 0 & 10 \\
Ischemia & 0 & 10 \\
* complication of esophageal cancer surgery, **fascitis of surgery site \\
Table 1. Frequency of causes of sepsis in both groups
\end{tabular}

\begin{tabular}{lccc}
\hline Variable & First group & Second group & P-value \\
\hline $\begin{array}{l}\text { Maximum heart } \\
\text { rate (per minute) }\end{array}$ & $119.9[12.8]$ & $127[8.3]$ & 0.39 \\
Maximum CVP(mm Hg) & $107.1-141.9$ & $115-145$ & \\
& $13.1[4.8]$ & $15.5[5.4]$ & 0.8 \\
Minimum CVP(mm Hg) & $9.5-22.7$ & $7.8-19.3$ & \\
& $8.8[3.8]$ & $6.8[5.1]$ & 0.25 \\
& $5.4-15.6$ & $3.2-12.8$ &
\end{tabular}




\begin{tabular}{llll}
\hline Maximum systolic & $133.4[44.5]$ & $138.2[14.2]$ & 0.63 \\
Blood pressure(mm Hg) & $116.4-172$ & $96.7-246$ & \\
Minimum systolic & $95[38.7]$ & $95.1[25.5]$ & 0.32 \\
Blood pressure(mm Hg) & $71.3-122.6$ & $55.6-108.1$ & \\
Total fluid volume & $4641[632.6]$ & $4845[880.1]$ & 0.22 \\
$\begin{array}{l}\text { During study(ml/day) } \\
\text { Total fluid volume }\end{array}$ & $2881.3-5526.3$ & $4081.3-6725$ & \\
$\begin{array}{l}\text { Outside the study } \\
\text { (ml/day) }\end{array}$ & $471.3[206]$ & $475.5[303.2]$ & 0.99 \\
Urine volume & $206.3-633.8$ & $175-637.5$ & \\
(mL/24hr) & & & \\
& $3578.5[1732.2]$ & $3907.3[1171.9]$ & 0.68 \\
APACHI II & $850-5365$ & $1055.6-6650$ & \\
& $21.8[7.5]$ & $21.2[2.7]$ & 0.80 \\
SOFA & $13.9-25.4$ & $10.9-29.6$ & \\
& $6.1[3.5]$ & $5.2[1.6]$ & 0.99 \\
& $2-8.9$ & $2-9.3$ &
\end{tabular}

Table 2. Variables during hospitalization in both groups

The variables during the hospitalization are listed in the table 2 . The median of maximum CVP in the first group was $13.1 \mathrm{~mm} \mathrm{Hg}$ and 15.5 $\mathrm{mm} \mathrm{Hg}$ in the second group $(p=0.8)$ and the median of minimum CVP was 8.8 in the first group and 6.8 in the second group $(p=0.25)$. The median of total fluid used in the study was $4641 \mathrm{ml} /$ day for the first group and 4845 $\mathrm{ml} /$ day for the second group $(p=0.22)$. The median of maximum systolic blood pressure was $133.4 \mathrm{~mm} \mathrm{Hg}$ in the first group and 138.2 in the second group $(p=0.63)$ and the median of minimum systolic blood pressure was $95 \mathrm{~mm}$ $\mathrm{Hg}$ in the first group and 95.1 in the second group $(p=0.32)$. Other variables are also listed in the table 2 and compared between the two groups. Serum levels of BUN and creatinine were within the normal range during the hospitalization. There was no need for dialysis for any of the patients during the study. The median of $\mathrm{PaO} 2$ and $\mathrm{O} 2$ sat in the second group was 93 [6] $\mathrm{mm} \mathrm{Hg}$ and 88 [4] percent and in the first group were 96 [5] $\mathrm{mm} \mathrm{Hg}$ and 91 [6] percent, respectively. Based on the MannWhitney $U$ test there was no significant difference between the two groups $(p=0.77$ and 0.45 respectively). 9 patients (90\%) in both groups needed mechanical ventilation, all eighteen had respiratory failure. The median duration of mechanical ventilation for the second group was 14 [19] days, with the minimum of 4 and maximum of 25 days and in the second group was 5 [17] days with the minimum of 3 and maximum of 13 days. There was no significant difference between the two groups based on the Mann-Whitney $U$ test $(p=0.24)$. The median duration of ICU hospitalization in the second group was 16 [21] 
days with the minimum of 7 and maximum of 40 days and 7.5 [5] days in the second group with the minimum of 4 and maximum of 35 days 9. Based on the Mann-Whitney $U$ test, there was a significant difference between the two groups. The median duration of ICU hospitalization in the second group was significantly higher than the first group $(p=$ 0.03). The median duration of hospitalization was 32 [28] days with the minimum of 16 and maximum of 53 days in the second group and 12 [6] days with the minimum of 9 and maximum of 18 days in the first group. The median duration of hospitalization was significantly higher in the second group than the first group ( $p=0.02$ ). In 28 days follow up, 1 patient in the second group (10\%) and 3 patients in the first group, expired and there was no significant difference between the two groups from this point of view, based on the fisher's exact test results $(p=0.58)$. In 90 days follow up, 4 patients in the second group (40\%) and 5 patients in the first group (50\%), expired. Based on the fisher's exact test results, there was no significant difference between the two groups $(p=0.50)$. The median time from hospitalization to decease for deceased cases was 39 [28] days with the minimum of 6 and maximum of 90 days in the second group and 20 [6] days with the minimum of 5 and maximum of 90 days in the first group. No significant difference found between the two groups based on the Mann-Whitney $U$ test results $(p=0.41)$.

\section{Discussion}

The 90 day mortality rate, detected in this study, as mentioned earlier was $40 \%$ in the second group and $50 \%$ in the first group and the difference between the two groups was not significant $(p=0.41)$. This range of mortality rate has also been reported in other literature; 24 $39 \%$ mortality rate in 28 days and $33-50 \%$ in 90 days follow up ${ }^{9-11}$. Navickis and Wilkes $(2001)^{12}$, gathered the results of the comparative studies of the administration of albumin and crystalloid fluids for ill patients and reached this conclusion that the administration of albumin does not decrease the mortality rate in these patients or decreases it insignificantly. From several aspects, the results of our study were concordant to this study. The difference between the two groups in terms of mortality rate, organ failure (based on the SOFA score) and dialysis status was not significant. Should be noted that none of the patients in our study needed dialysis. However, the median duration of hospitalization in ICU and non-ICU in the second group was significantly higher than the first group (16 vs. 7.5 and 32 vs. 12 days, respectively).

Administration of albumin, increased the median of duration of need for mechanical ventilation (14 vs. 5 days), but the difference was not significant between the two groups. Patel et al (2014) ${ }^{13}$, reviewed the results of albumin administration from 18 clinical trial studies, including 4190 patients, with different degrees of sepsis. During 3 days, approximately 70 grams of albumin were administered and no significant difference was seen between the two groups from the mortality point of view ( $R R=0.94) ; \quad$ they concluded that the administration of albumin does not decrease the mortality rate.

In a random clinical trial conducted by Caironi et al $(2014)^{8}, 1818$ patients with severe sepsis in 100 intensive care units, randomly 
underwent the administration of $20 \%$ albumin or crystalloid serums; there was no significant difference between the two groups from 28 and 90 day mortality rate and organ failure point of view, from which results they concluded that the administration of albumin has no benefit for the patients with severe sepsis. Align with the results of this study, we also didn't find any significant difference between the two groups in mortality rate and organ failure. Chang and Holcomb (2016) ${ }^{14}$, concluded that the outcomes of administration of albumin compared to crystalloid fluids, are similar and due to the of albumin being more expensive, it is better to use only crystalloid fluids for the patients with severe sepsis and septic shock.

Dubois et al $(2006)^{15}$, The SAFE Study Investigators $(2006)^{16}$ and Goldwasser and Feldman $(1997)^{17}$ suggest that the administration of albumin only improves organ function and should only be used for adjustment of hypoalbuminemia in the patients with severe sepsis. Although, we didn't include the cost of the intervention, but regarding the high cost of albumin compared to crystalloid fluids and increased duration of non-ICU hospitalization with the administration of albumin, which can cause extra cost and hospitalization related complications in the patients, however, with nonsignificant decrease in mortality rate, we do not suggest the administration of albumin for these patients, which is aligned with the three latter studies mentioned above. Since we didn't include the cost, further cost-effectiveness studies may be of benefits.

The SAFE Study Investigators $(2011)^{6}$, compared the mortality and organ failure after the administration albumin and saline, in 1,218 patients with severe sepsis. They suggest that the administration of albumin does not impair the function of any organ compared to saline but it may decrease the mortality. The results of our study align with these results except that in our study, the mortality rate between the two groups had no significant difference.

The results of a meta analysis conducted by Delaney et al $(2011)^{7}$, suggest that the administration of albumin can significantly decrease the mortality $(p=0.05, O R=0.82)$. In another meta analysis, $\mathrm{Xu}$ et al $(2014)^{18}$, reached to this conclusion that the 90 day mortality rate in the patients with severe sepsis who received albumin, tend to decrease more compared to patients receiving crystalloid fluids ( $p=0.08, O R=0.88)$, but this decrease was significant in the patients with septic shock $(p=0.03, O R=0.81)$. Unlike this study, we didn't find any significant difference between the two groups in terms of mortality and prognosis and against the results of this study, we found that the administration of crystalloid fluids, decreases the median duration of ICU and nonICU hospitalization, significantly, compared to albumin.

One of the reasons for supporting the albumin as a natural colloid in the patients with septic shock, is the increase in the colloid osmotic pressure and CVP and decrease in heart rate in these patients compared to the crystalloid receiving patients ${ }^{8,19-23}$. Dellinger et al $(2013)^{24}$, concluded that the administration of albumin, alongside the crystalloid fluids, can be helpful in maintaining the effective volume and establishing the colloid osmotic pressure in the patients with severe sepsis and septic shock. Trof et al $(2010)^{25}$, suggest that in these 
patients, the use of albumin compared to normal saline, provides greater cardiac responses.

Some theories, support the thesis of the need for albumin in the patients with severe sepsis and septic shock, which are listed here ${ }^{1,26-29}$ :

1. Albumin is the main protein that produces the colloid osmotic pressure in plasma

2. Albumin acts as a transporter for several endogenous and exogenous compounds

3. Albumin has an antioxidant and anti inflammatory role as the neutralizer of reactive oxygen and nitrogen species

4. Albumin acts as a buffer molecule to regulate the acid base balance
The common point in these studies, is that, in the patients with severe sepsis or septic shock, the fluid balance is of vital importance ${ }^{30-32}$. The most important limitation in our study was the sample size being small, but it's better be noted that our study was a pilot trial for planning to conduct further large studies. Since adding albumin to the crystalloid fluids, increased the duration of ICU and non-ICU hospitalization without any improvements in the prognosis, it is not recommended. Although, in order to reach to certain results, further studies with larger sample sizes, different degrees of sepsis and septic shock simultaneously, longer followups and considering cost-effectiveness of albumin seem to be needed.

\section{References}

1. Quinlan GJ, Martin GS, Evans TW. (2005). Albumin: biochemical properties and therapeutic potential. Hepatology, 41 (6), 1211-1219.

2. Horsey PJ. (2002). The Cochrane 1998 Albumin Review--not all it was cracked up to be. Eur J Anaesthesiol, 19 (10), 701-704.

3. Quinlan GJ, Mumby S, Martin GS, Bernard GR, Gutteridge JM, Evans TW. (2004). Albumin influences total plasma antioxidant capacity favorably in patients with acute lung injury. Crit Care Med, 32(3), 755-759.

4. Schwartz SI, Brunicardi FC (2010). Schwartz's principles of surgery, $9^{\text {th }}$ ed. McGraw-Hill, Medical Pub. Division, New York, 1012-1015.

5. Leitch A, Craig G, Sadler P. (2013). Human Albumin Solution Resuscitation in Severe Sepsis and Septic Shock. Journal of the Intensive Care Society, 14(1), 45-52.

6. The SAFE Study Investigators Intensive Care Med (2011) 37: 86. doi:10.1007/s00134-010-2039-6

7. Delaney AP, Dan A, McCaffrey, J, Finfer S. (2011). The role of albumin as a resuscitation fluid for patients with sepsis: a systematic review and metaanalysis. Crit Care Med, 39(2), 386-391.

8. Caironi P, Tognoni G, Masson S, Fumagalli R, Pesenti A, Romero M, et al. (2014). Albumin replacement in patients with severe sepsis or septic shock. N Engl J Med, 370(15), 1412-1421.

9. Vincent JL, Sakr Y, Sprung CL, Ranieri VM, Reinhart K, Gerlach H, et al. (2006). Sepsis in European intensive care units: results of the SOAP study. Crit Care Med, 34(2), 344-353.

10. Ranieri VM, Thompson BT, Barie PS, Dhainaut JF, Douglas IS, Finfer S, et al. (2012). Drotrecogin alfa (activated) in adults with septic shock. N EnglJ Med, 366(22), 2055-2064.

11. Russell JA, Walley KR, Singer J, Gordon AC, Hebert PC, Cooper DJ, et al. (2008). Vasopressin versus norepinephrine infusion in patients with septicshock. N Engl J Med, 358(9), 877-887. 
12. Wilkes MM, Navickis RJ. (2001). Patient survival after human albumin administration. A meta-analysis of randomized, controlled trials. Ann Intern Med, 135(3), 149-164.

13. Patel A, Laffan MA, Waheed U, Brett SJ. (2014). Randomised trials of human albumin for adults with sepsis: systematic review and meta-analysis with trial sequential analysis of all-cause mortality. BMJ, 349g4561.

14. Chang R, Holcomb JB. (2016). Choice of Fluid Therapy in the Initial Management of Sepsis, Severe Sepsis, and Septic Shock. Shock.

15. Dubois MJ, Orellana-Jimenez C, Melot C, De Backer D, Berre J, Leeman M, et al. (2006). Albumin administration improves organ function incritically ill hypoalbuminemic patients: A prospective, randomized,controlled, pilot study. Crit Care Med, 34(10), 2536-2540.

16. The SAFE Study Investigators (2006). Effect of baseline serum albumin concentration on outcome of resuscitation with albumin or saline in patients in intensive care units: analysis of data from the saline versus albumin fluid evaluation (SAFE) study. BMJ, 333(7577), 1044.

17. Goldwasser P, Feldman J. (1997). Association of serum albumin and mortality risk. J Clin Epidemiol, 50(6), 693-703.

18. Xu JY, Chen QH, Xie JF, Pan C, Liu SQ, Huang LW, et al. (2014). Comparison of the effects of albumin and crystalloid on mortality in adult patients with severe sepsis and septic shock: a meta-analysis of randomized clinical trials. Crit Care, 18(6), 702.

19. Friedman G, Jankowski S, Shahla M, Gomez J, Vincent JL. (2008). Hemodynamic effects of $6 \%$ and $10 \%$ hydroxyethyl starch solutions versus 4\% albumin solution in septic patients. J Clin Anesth, 20(7), 528-533.

20. Rackow EC, Falk JL, Fein IA, Siegel JS, Packman MI, Haupt MT, et al. (1983). Fluid resuscitation in circulatory shock: a comparison of the cardiorespiratory effects of albumin, hetastarch, and saline solutions in patients with hypovolemic and septic shock. Crit Care Med, 11(11), 839-850.

21. van der Heijden M, Verheij J, van Nieuw Amerongen GP, Groeneveld AB. (2009). Crystalloid or colloid fluid loading and pulmonary permeability, edema, and injury in septic and nonseptic critically ill patients with hypovolemia. Crit Care Med, 37(4), 1275-1281.

22. Dolecek M, Svoboda P, Kantorova I, Scheer P, Sas I, Bibrova J, et al. (2009). Therapeutic influence of $20 \%$ albumin versus $6 \%$ hydroxyethylstarch on extravascular lung water in septic patients: a randomized controlled trial. Hepatogastroenterology, 56(96), 1622-1628.

23. Trof RJ, Sukul SP, Twisk JW, Girbes AR, Groeneveld AB. (2010). Greater cardiac response of colloid than saline fluid loading in septic and non-septic critically ill patients with clinical hypovolaemia. Intensive Care Med, 36(4), 697-701.

24. Dellinger RP, Levy MM, Rhodes A, Annane D, Gerlach H, Opal SM, et al. (2013). Surviving sepsis campaign: international guidelines for management of severe sepsis and septic shock: 2012. Crit Care Med, 41(2), 580637.

25. Trof RJ, Sukul SP, Twisk JW, Girbes AR, Groeneveld AB. (2010). Greater cardiac response of colloid than saline fluid loading in septic and non-septic critically ill patients with clinical hypovolaemia. Intensive Care Med, 36(4),697-701.

26. Weil MH, Henning RJ, Puri VK. (1979). Colloid oncotic pressure: clinical significance. Crit Care Med, 7(3), 113116.

27. Sudlow G, Birkett DJ, Wade DN. (1975). The characterization of two specific drug binding sites on human serum albumin. Mol Pharmacol, 11(6), 824-832.

28. Quinlan GJ, Margarson MP, Mumby S, Evans TW, Gutteridge JM. (1998). Administration of albumin to patients with sepsis syndrome: a possible beneficial role in plasma thiol repletion. Clin Sci (Lond), 95(4), 459465.

29. Stamler JS, Jaraki O, Osborne J, Simon DI, Keaney J, Vita J, et al. (1992). Nitric oxide circulates in mammalian plasma primarily as an S-nitroso adduct of serum albumin. Proc Natl Acad Sci U S A, 89(16), 7674-7677. 
30. Pro Cl, Yealy DM, Kellum JA, Huang DT, Barnato AE, Weissfeld LA, et al. (2014). A randomized trial of protocolbased care for early septic shock. N Engl J Med, 370(18), 1683-1693.

31. Rivers E, Nguyen B, Havstad S, Ressler J, Muzzin A, Knoblich B, et al. (2001). Early goal-directed therapy in the treatment of severe sepsis and septic shock. N Engl J Med, 345(19), 1368-1377.

32. Lilly CM. (2014). The ProCESS trial--a new era of sepsis management. N Engl J Med, 370(18), 1750-1751. 